\title{
EFFECTS OF INLET GUIDE VANES ON THE PERFORMANCE AND STABILITY OF AN AERONAUTICAL CENTRIFUGAL COMPRESSOR
}

\author{
Nicolas Poujol *,1,2, Isabelle Trébinjac², Pierre Duquesne ${ }^{2}$ \\ 1Safran Helicopter Engines, Bordes, France \\ 'Laboratoire de Mécanique des Fluides et d'Acoustique, Ecole Centrale de Lyon, Ecully, France
}

\section{ABSTRACT}

A research centrifugal compressor stage designed and built by Safran Helicopter Engines is tested at 3 IGV (Inlet Guide Vanes) stagger angles. The compressor stage includes 4 blade rows: axial inlet guide vanes, a backswept splittered impeller, a splittered vaned radial diffuser and axial outlet guide vanes. The methodology for calculating the performance is detailed, including the consideration of humidity in order to minimize errors related in particular to operating atmospheric conditions.

The shift of the surge line towards lower mass flow rate as the IGV stagger angle increases highly depends on the rotation speed. The surge line shift is very small at low rotation speeds whereas it significantly increases at high rotation speeds. A firstorder stability analysis of the impeller and diffuser subcomponents shows that the diffuser (resp. impeller) is the first unstable component at low (resp. high) rotation speeds. This situation is unaltered by increasing the IGV stagger angle.

At low rotation speeds below a given mass flow rate, rotating instabilities at the impeller inlet are detected at zero IGV stagger angle. Their occurrence is conditioned by the relative flow angle at the tip of the leading edge of the impeller. As the $I G V$ stagger angle increases, the mass flow decreases to maintain a given inlet flow angle. Therefore, the onset of the rotating instabilities is delayed towards lower mass flow rates.

At high rotation speeds, the absolute flow angle at the diffuser inlet near surge decreases as the IGV stagger angle increases. As a result, the flow is highly alternate over two adjacent channels of the radial diffuser beyond the surge line at IGV stagger angle of $0^{\circ}$.

Keywords: centrifugal compressor, inlet guide vanes, rotating instabilities, surge

\section{INTRODUCTION}

Environmental issues and the market environment encourage the engine manufacturers to improve the versatility of helicopter turboshafts. Therefore, the operating range of compressors should be extended while maintaining a high level of efficiency. There are two ways to extend the operating range of compressors:

- Reduce the surge margin by understanding better the local instabilities that cause deep surge.
However, some complex instabilities such as spikes are still poorly understood [1] thus this method might not work in all cases for coming years. It should also be noted that the surge margin would reach a minimal value due to production tolerance and ageing of engines [2],

- Push towards lower mass flow rates the onset of deep surge and local instabilities. This goal might be achieved by inserting technology effects such as casing treatment, variable Inlet Guide Vanes (IGV) and boundary layer suction [3, 4]. The main difficulty of this method is to maintain the high level of efficiency at the design point while pushing towards lower mass flow rates the onset of deep surge.

The second method with the use of IGV for a centrifugal compressor is studied in the present work. Indeed, Whitfield et al. [5] have shown that the positive (in the direction of rotation) inlet pre-swirl pushed the onset of local instabilities, recirculation and deep surge towards lower mass flow rates. An inlet pre-swirl can be achieved in helicopter engines with IGV which have been previously used in numerous centrifugal compressor applications: Auxiliary Power Unit (APU) [6], terrestrial industrial compressor [7] or turbocharger [8].

The IGV can be set at different stagger angles to modify the incidence at impeller inlet. Tian et al. [9] noticed that the preswirl angle was lower than the IGV stagger angle. This lag effect has been attributed to the large IGV hub and the flow separation. Positive inlet pre-swirl induced by the IGV affect nevertheless the range of high efficiency and the stability of the compressor. The experimental and numerical investigations of Mohseni et al. [10] point to an extension of the range of high efficiency for IGV with s-cambered profiles and positive pre-swirl for stagger angles up to $+75^{\circ}$. Experimental work of Rodgers [6] at different stagger angles indicates that the onset of the deep surge is pushed towards lower mass flow rates at high rotation speeds with a positive pre-swirl even if the radial diffuser is stalled. The use of IGV for a turbocharger has been studied by Uchida et al. [11]. The IGV stagger angles has been set up to $+80^{\circ}$ and the surge line has been shifted towards lower mass flow rates up to $15 \%$. Ishino et al. [8] observed that the deviation caused by the IGV

*Corresponding author: nicolas.poujol@ec-lyon.fr 
reduces the recirculation occurring between the suction side of the inducer and the shroud.

Most of the works cited above study the effect of the IGV stagger angle at only one rotation speed. The present work aims at investigating the effect of the IGV stagger angle over the complete performance map. Furthermore, the effect of IGV on the development of instabilities is rarely investigated. In particular, none work on the impact of the IGV stagger angle on the onset of rotating instabilities in centrifugal compressors has been published to the authors' knowledge. Therefore, the present work also focuses on the impact of the IGV stagger angle on the onset of rotating instabilities.

First, the test case, the experimental setup and the performance calculation methodology are described. The performance maps of the compressor stage for three IGV stagger angles are given and discussed. Then, the impact of the IGV stagger angle on the onset of rotating instabilities is studied. Finally, the evolution of the flow topology in the radial diffuser according to the IGV stagger angle is analyzed.

\section{TEST CASE}

The test case is a research centrifugal compressor stage designed and manufactured by Safran Helicopter Engines. The compressor stage includes four blade rows: axial inlet guide vanes (IGV), a backswept splittered impeller (IMP.), a splittered vaned radial diffuser (RD) and axial outlet guide vanes (OGV). A meridional scheme of the compressor is provided in Fig. 1.

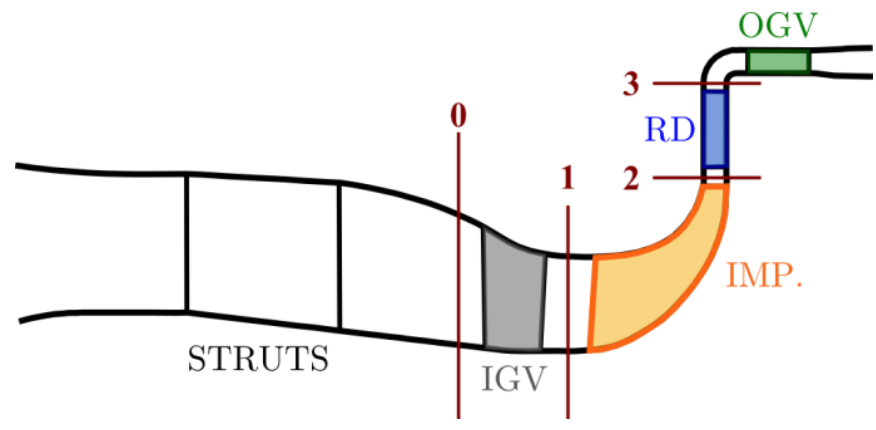

FIGURE 1: MERIDIONAL VIEW OF THE COMPRESSOR

The overall stage total to static pressure ratio is around 4 . The Mach number based on the peripheral velocity at the exit radius of the impeller is around 1.2.

\section{EXPERIMENTAL SETUP AND PERFORMANCE CALCULATION}

The centrifugal compressor stage is mounted on the $1 \mathrm{MW}$ test rig of Ecole Centrale de Lyon and is considerably instrumented. More than a hundred steady pressure measurements and almost 20 thermocouples are used for the monitoring and the performance measurements. The mass flow is measured with a diaphragm flowmeter which is located at the test rig inlet.

The distribution of the steady pressure sensors in two channels of the radial diffuser is given in Fig. 2. Indeed, a particular flow topology spread on two channels of the radial diffuser has been previously observed numerically [12] and experimentally [13] at an IGV stagger angle of $0^{\circ}$. The impact of the IGV stagger angle on this alternate flow topology is studied in the last part of the paper. The steady pressure sensors are located at the shroud or/and the hub.

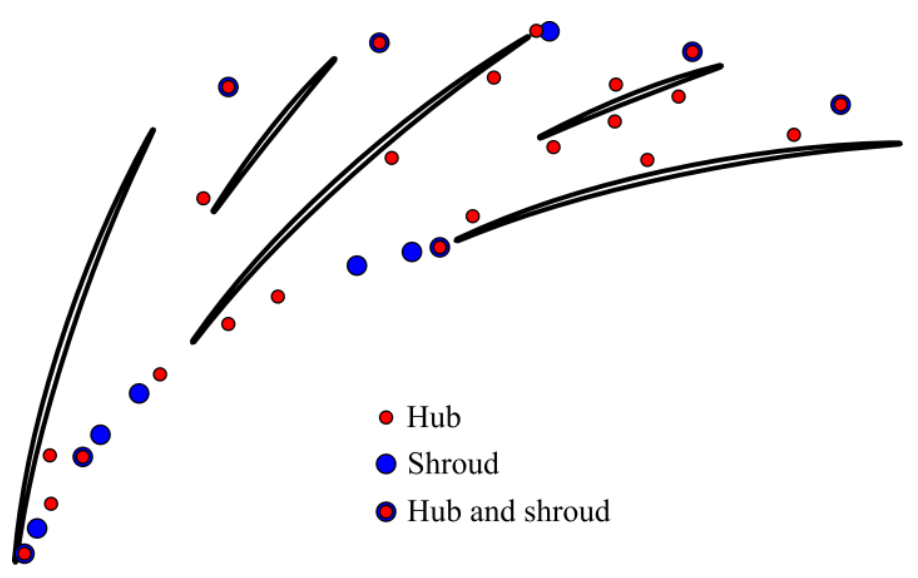

FIGURE 2: DISTRIBUTION OF THE STEADY PRESSURE SENSORS IN TWO CHANNELS OF THE RADIAL DIFFUSER

Furthermore, 54 unsteady pressure sensors (models XTE190M and XTEH-7L-190M manufactured by Kulite Semiconductor Product Inc.) are distributed over the entire stage in order to capture the time-dependent fluctuations of the flow and to investigate instabilities. To capture phenomena at frequencies around $100 \mathrm{kHz}$, the protection grids were removed in order to maximize the natural frequency of the probes. The average value of the uncertainties of the fluctuations is less than $0.7 \%$ of the peak-to-peak pressure fluctuation of the signal.

Ten unsteady pressure sensors are circumferentially distributed at the shroud at the impeller inlet ( $1 \mathrm{~mm}$ upstream the impeller leading edge), the distribution is provided in Fig. 3. Moreover, 5 unsteady pressure sensors are distributed along the external meridional path from the impeller leading edge to the impeller trailing edge. There are also 36 unsteady pressure sensors in the radial diffuser whose the location is not given because they are not used in the following analysis. 


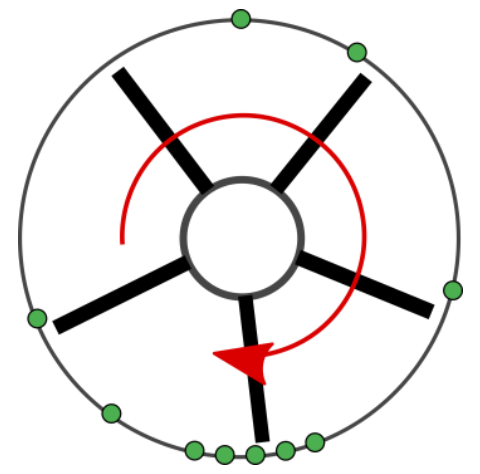

FIGURE 3: DISTRIBUTION OF THE UNSTEADY PRESSURE SENSORS AT THE IMPELLER INLET

For each stagger angle, the performance map of the compressor stage is explored by increasing the rotation speed. At each rotation speed, the compressor is throttled by around 15 increments from choke to surge in order to obtain a fine discretization of the performance characteristic. The throttling increments are reduced when moving towards surge in order to accurately determine the surge mass flow rate. Deep surge is detected both by measurement of the temperature $T_{t, 0}$ (thermocouple upstream the IGV row) increase and by its associated noise heard in the control room.

At each throttling value, around 5 minutes are needed to reach the thermal stability of the compressor. When the stability is reached, the steady measurements are recorded and averaged during 15 seconds in order to reduce the measurement uncertainties. The measurement uncertainties at the design point are given in Tab. 1. The systematic uncertainties are considered to be negligible, only the random uncertainties are presented here.

TABLE 1: STEADY MEASUREMENT UNCERTAINTIES AT THE DESIGN POINT

\begin{tabular}{|c|c|}
\hline Measured value & Uncertainty \\
\hline$\Omega$ & $\pm 0.06 \%$ \\
\hline$P$ & $\pm 0.01 \%$ \\
\hline$T$ & $\pm 0.20 \%$ \\
\hline
\end{tabular}

The rotation speed of the compressor is determined during the measurements by the standard rotation speed targeted and the atmospheric conditions of the day including the relative humidity (as explained later).

$$
\Omega=\frac{\sqrt{\gamma r T_{t, 0}}}{\sqrt{\gamma_{r e f} r_{r e f} T_{r e f}}} \Omega_{s t d}
$$

The performance values of the compressor stage are calculated with the Eq. 2, Eq.3 and Eq. 4.

$$
\dot{m}_{s t d}=\frac{\sqrt{r \gamma_{r e f} T_{t, 0}}}{\sqrt{r_{r e f} T^{T} T_{r f}}} \frac{P_{r e f}}{P_{t, 0}} \dot{m}
$$

The outlet guide vanes are not taken into account for the performance estimation of the compressor stage: the performance values of the compressor are calculated between the planes 3 and 0 in Fig. 1.

$$
\begin{aligned}
& \pi_{C}^{t-s}=\frac{P_{s, 3}}{P_{t, 0}} \\
& \eta_{C}=\frac{T_{t, 0}\left(\pi_{C}^{t-s^{\frac{\gamma-1}{\gamma}}-1}\right)}{T_{t, 3}-T_{t, 0}}
\end{aligned}
$$

There are several steady pressure and temperature sensors distributed all around the circumference at each reference plane in order to reduce measurement errors: the values are averaged at each reference plane. The performance of the components is calculated with the same methodology.

The uncertainties on the performance values are calculated with propagation formulas and given at the design operating point in Tab. 2 .

TABLE 2: MEASUREMENT UNCERTAINTIES OF PERFORMANCE VALUES AT THE DESIGN POINT

\begin{tabular}{|c|c|}
\hline Performance value & Uncertainty \\
\hline$\dot{m}$ & $\pm 0.11 \%$ \\
\hline$\pi_{C}{ }^{t-s}$ & $\pm 0.13 \%$ \\
\hline$\eta_{c}$ & $\pm 0.21 \%$ \\
\hline
\end{tabular}

The uncertainties on performance values at the design point are low. It should be noted that for lower mass flow rates (especially at low rotation speeds near surge), the uncertainty on mass flow rate can reach up to $0.4 \%$ for example. However, this measurement accuracy is more than sufficient for quantifying the impact of the stagger angle of the IGV on the performance values of the compressor stage.

The specific heat ratio $\gamma$ and the specific gas constant $r$, appearing in Eq. 1, Eq. 2 and Eq.4, depend on the humidity rate of the day. Therefore, the humidity rate might be considered in order to minimize errors related in particular to operating atmospheric conditions. A humidity sensor is positioned in the settling chamber where the total temperature and total pressure are also measured. The empirical relation found by Glück [14] is used to calculate the saturation vapor pressure with the total temperature at the inlet of the compressor. Then, the water vapor concentration in the flow is straightforward calculated and the values of the specific heat ratio $\gamma$ and specific gas constant $r$ are deduced.

These two specific values ( $\gamma$ and $r$ ) of the flow are used at two times (as shown in Fig. 4):

- During measurements for determining the rotation speed in order to be consistent with the standard rotation speed,

- After measurements, for the performance calculation. 


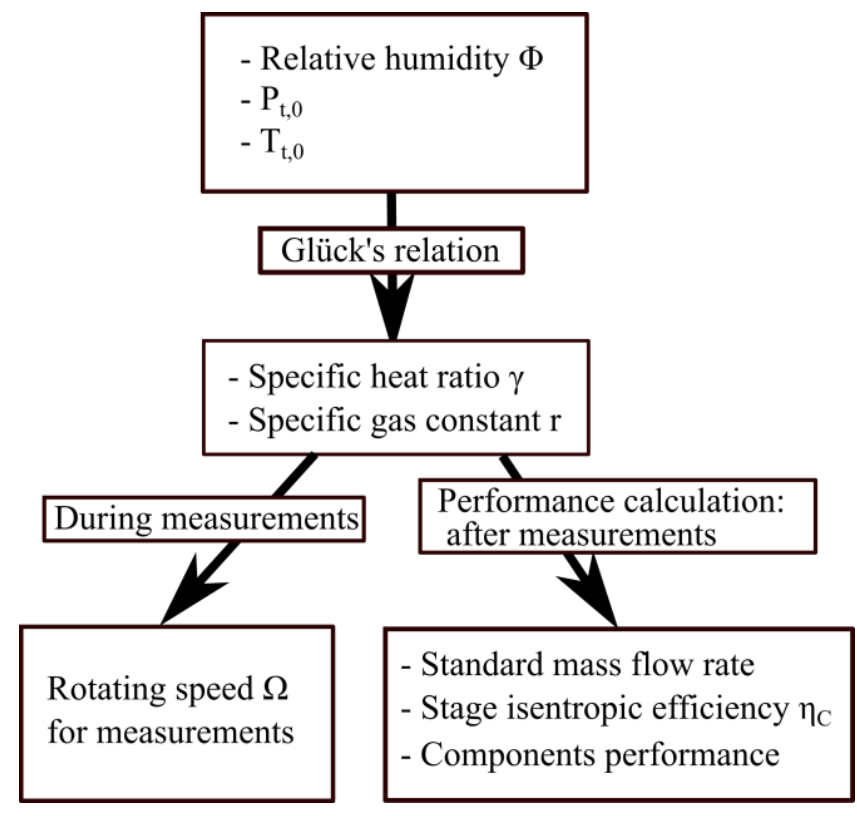

FIGURE 4: METHODOLOGY OF INCLUSION OF HUMIDITY RATE FOR MEASUREMENTS

The additional error of characteristic values, at the design operating point, calculated without the inclusion of humidity rate are given in Table 3. It should be noted that these errors depend on the atmospheric conditions of the day $(\Phi=55 \%$ for these measurements).

TABLE 3: PERFORMANCE ERRORS WITHOUT THE INCLUSION OF HUMIDITY RATE

\begin{tabular}{|c|c|}
\hline Characteristic value & Additional error \\
\hline$\Omega$ & $0.7 \%$ \\
\hline$\dot{m}_{s t d}$ & $0.01 \%$ \\
\hline$\eta_{c}$ & $1.11 \%$ \\
\hline
\end{tabular}

The error on the standard mass flow rate is negligible but the errors on the rotation speed and on the stage isentropic efficiency are significant. Indeed, the error on the isentropic efficiency is about 5 times higher than the measurement uncertainty. It highlights that the humidity rate has to be measured and taken into account a priori (during measurements to maintain the correct standard rotation speed) and a posteriori (for the performance calculation).

\section{PERFORMANCE MAP AND VARIATION OF THE CHOKED MASS FLOW RATE}

Previous work on the compressor at an IGV stagger angle of $0^{\circ}$ has been carried out by Moënne-Loccoz et al. [13, 15]. Rotating instabilities at the impeller inlet have been observed, the impact of the IGV stagger angle on the development of these rotating instabilities is discussed in the last part.
The performance map of the compressor stage has been measured at 7 rotation speeds from choke to surge for 3 IGV stagger angles: $0^{\circ}, 20^{\circ}$ and $40^{\circ}$ (positive pre-swirl). It can be noted that the IGV clearances increase at both ends of the vane as the IGV stagger angle increases. From 11 to 21 stabilized operating points have been captured for each operating speed and for each stagger angle. Therefore, the performance map is well discretized. Rotation speeds can be divided into two groups: low rotation speeds (L1 to L3) and high rotation speeds (H1 to H4). Therefore only a portion of the performance map with two rotation speeds (each representative of one group) is given in Fig. 5 for confidentiality reasons. The measurement uncertainties are lower than the marker size, therefore non visible on Fig. 5. The surge line is plotted for each stagger angle in thick lines on Fig. $5 \mathrm{a}$.

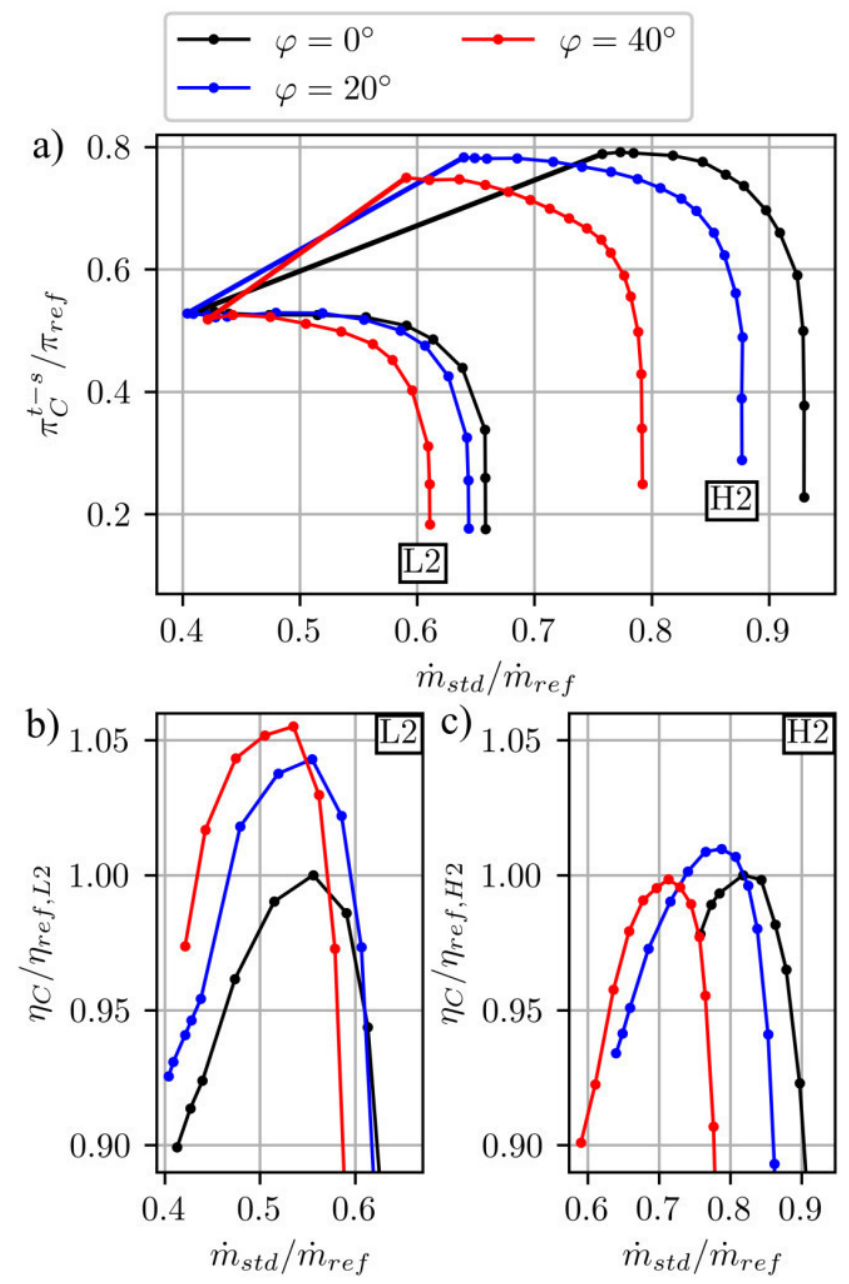

FIGURE 5: PORTION OF THE PERFORMANCE MAP AT 3 STAGGER ANGLES $\varphi$ 
As the stagger angle increases (for positive pre-swirl) the curves are shifted towards lower mass flow rates and slightly lower pressure ratios. However, the curves shift highly depends on the rotation speed, for example:

- At the low rotation speed L2, the stagger angle has a small impact on the pressure ratio curve.

- At the high rotation speed $\mathrm{H} 2$, the increase of the stagger angle shifts considerably the pressure ratio curve.

At low rotation speeds, the compressor stage reaches the same pressure ratio as the stagger angle increases contrary to the high rotation speeds especially at the stagger angle of $40^{\circ}$.

The stage isentropic efficiency is normalized by the maximum efficiency for the rotation speed considered at stagger angle of $0^{\circ}$. The stage isentropic efficiency is considerably increased at low rotation speeds as the stagger angle increases (see Fig. 5 b). In addition, the mass flow rate of the peak efficiency operating point is almost unaltered by the stagger angle at low rotation speeds. In contrast, the stage isentropic efficiency is slightly modified by the stagger angle at high rotation speeds but the mass flow rate of the peak efficiency operating point is greatly affected (see Fig. 5 c). Therefore, the mass flow range of high efficiency is considerably extended by adjusting the IGV stagger angle.

At a given rotation speed, the operating range is limited on the one hand by the choked mass flow and on the other hand by the surge mass flow. The impact of the stagger angle on the choked mass flow and the shift of the surge line towards lower mass flow rates are now being analyzed. The choked mass flow rate is considerably reduced as the stagger angle increases. The change in choked mass flow rate at a given rotation speed is quantified (with $\varphi_{\mathrm{a}}$ and $\varphi_{\mathrm{b}}$ two different stagger angles) by:

$$
\Delta \dot{m}_{s t d}^{\text {choke }}\left(\varphi_{b} V S \varphi_{a}\right)=\left|\dot{m}_{s t d, b}^{\text {choke }}-\dot{m}_{s t d, a}^{\text {choke }}\right|
$$

Fig. 6 shows the results for the 7 rotation speeds explored, the $\mathrm{y}$-axis being $\Delta \dot{m}_{s t d}^{\text {choke }}\left(\varphi_{b} V S \varphi_{a}\right)$ and the $\mathrm{x}$-axis being $\dot{m}_{s t d, a}^{\text {choke }}$. The rotation speeds $\mathrm{H} 3$ and $\mathrm{H} 4$ have not been explored at the stagger angle of $40^{\circ}$ which explains that the range in mass flow rate of the two curves is different.

The difference of choked mass flow rates is higher between $40^{\circ}$ and $20^{\circ}$ than $20^{\circ}$ and $0^{\circ}$ for all rotation speeds (the red curve is always above the blue one). The effect of the stagger angle on the choked mass flow is indeed not linear.

In addition, the curves diverge: the difference $\Delta \dot{m}_{\text {std }}^{\text {choke }}\left(\varphi_{b} v s \varphi_{a}\right)$ increases as the choked mass flow increases, i.e. as the rotation speed increases. Therefore, these curves illustrate the horizontal shift of the choked mass flow rate as a function of the stagger angle in Fig. 5.

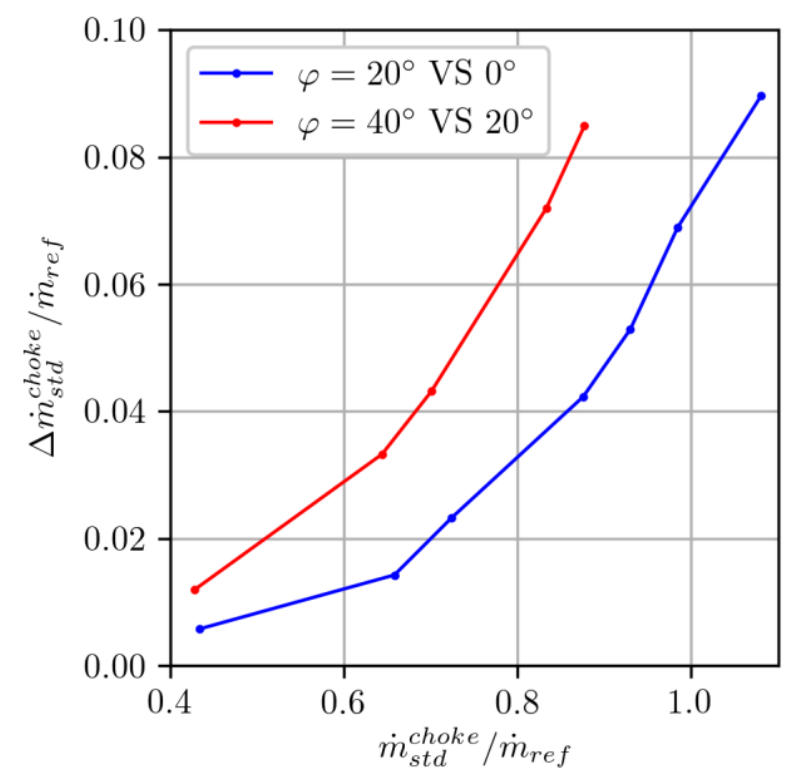

FIGURE 6: DIFFERENCE IN CHOKED MASS FLOW DUE TO A STAGGER ANGLE INCREASE

The second limit of the operating range is the surge mass flow rate which depends on the incidence on the impeller amongst other parameters. The velocity triangles at the impeller inlet are determined, assuming that the flow at the impeller inlet is aligned with the IGV trailing edge. Such an assumption has been evaluated from numerical simulations RANS run with the elsA software from ONERA. The simulations are run on the whole stage (including the OGV row) with mixing planes between the stationary and rotating components. As these simulations are only used to assess the validity of the previous assumption, further details on the parameters are not given. However, it should be noted that the parameters recommended by the ONERA are used.

The absolute flow angle at the IGV outlet (before the mixing plane between the IGV row and the impeller) is extracted for a numerical operating point near the experimental operating point corresponding to the onset of rotating instabilities. The results are that (i) the value of the average of the absolute flow angle does not depend on the rotation speed for a given stagger angle, (ii) the difference between the IGV stagger angle and the absolute flow angle is similar between all stagger angles and very low (less than $4^{\circ}$ at the worst case). Therefore, the differences are sufficiently small to validate the assumption. Furthermore, this assumption was done in order to establish a method only using experimental data.

Two velocity triangles at the impeller inlet at a same rotation speed and same mass flow rate are given in Fig. 7: without pre-swirl in black, with a pre-swirl of $+40^{\circ}$ in red. 


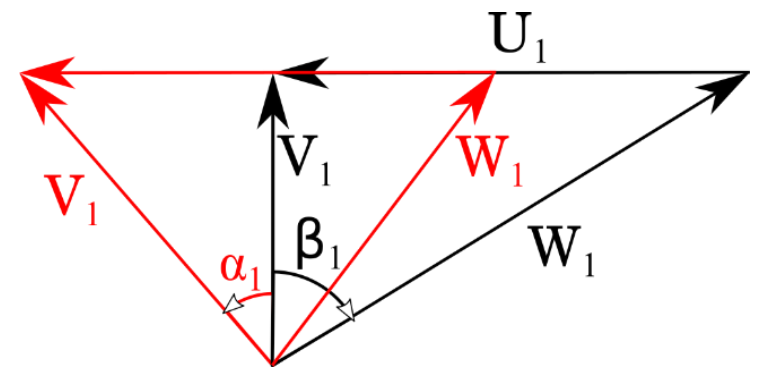

\section{FIGURE 7: VELOCITY TRIANGLES AT IMPELLER INLET}

The incidence at impeller inlet at a same mass flow rate is thus reduced by increasing the IGV stagger angle especially near deep surge. Therefore, increasing the IGV stagger angle might extend the operating range towards lower mass flow rates.

\section{ANALYSIS OF THE STABILITY OF COMPONENTS}

A first-order stability analysis of the components (based on Dean's work [16]) is performed. A compression system is defined stable if the slope of the total-to-static pressure ratio with the mass flow rate is negative:

$$
\frac{\partial \pi_{C}^{t-s}}{\partial \dot{m}_{s t d}} \leq 0
$$

One can split the pressure ratio of the compressor in the product of the pressure ratios of each component. By applying a logarithmic derivative, the stability parameter of each component appears:

$$
\underbrace{\frac{1}{\pi_{C}^{t-s} \frac{\partial \pi_{C}^{t-s}}{\partial \dot{m}_{s t d}}}}_{S P_{C}}=\underbrace{\frac{1}{\pi_{I G V}^{t-t} \frac{\partial \pi_{I G V}^{t-t}}{\partial \dot{m}_{s t d}}}}_{S P_{I G V}}+\underbrace{\frac{1}{\pi_{R}^{t-s} \frac{\partial \pi_{R}^{t-s}}{\partial \dot{m}_{s t d}}}}_{S P_{R}}+\underbrace{\frac{1}{\pi_{D R}^{s-s} \frac{\partial \pi_{D R}^{S-s}}{\partial \dot{m}_{s t d}}}}_{S P_{D R}}
$$

The stability parameter is computed with an off-centered derivative to the right. The stability parameter uncertainties are calculated using propagation formulas and plotted with error bars. For the last stable operating point, the uncertainties cannot be computed, the value plotted is the same as the penultimate stable operating point. The stability parameters of the whole compressor stage, the impeller and the radial diffuser are computed. The IGV stability parameter is not studied because it is always stable due to pressure losses. Indeed, pressure losses being proportional to the square of the mass flow rate, the total to total pressure ratio of the IGV decreases as the mass flow rate increases.
The stability parameters for a stagger angle of $0^{\circ}$ for all rotation speeds explored are given in Fig. 8. The error bars are smaller than the marker size for all presented operating points.
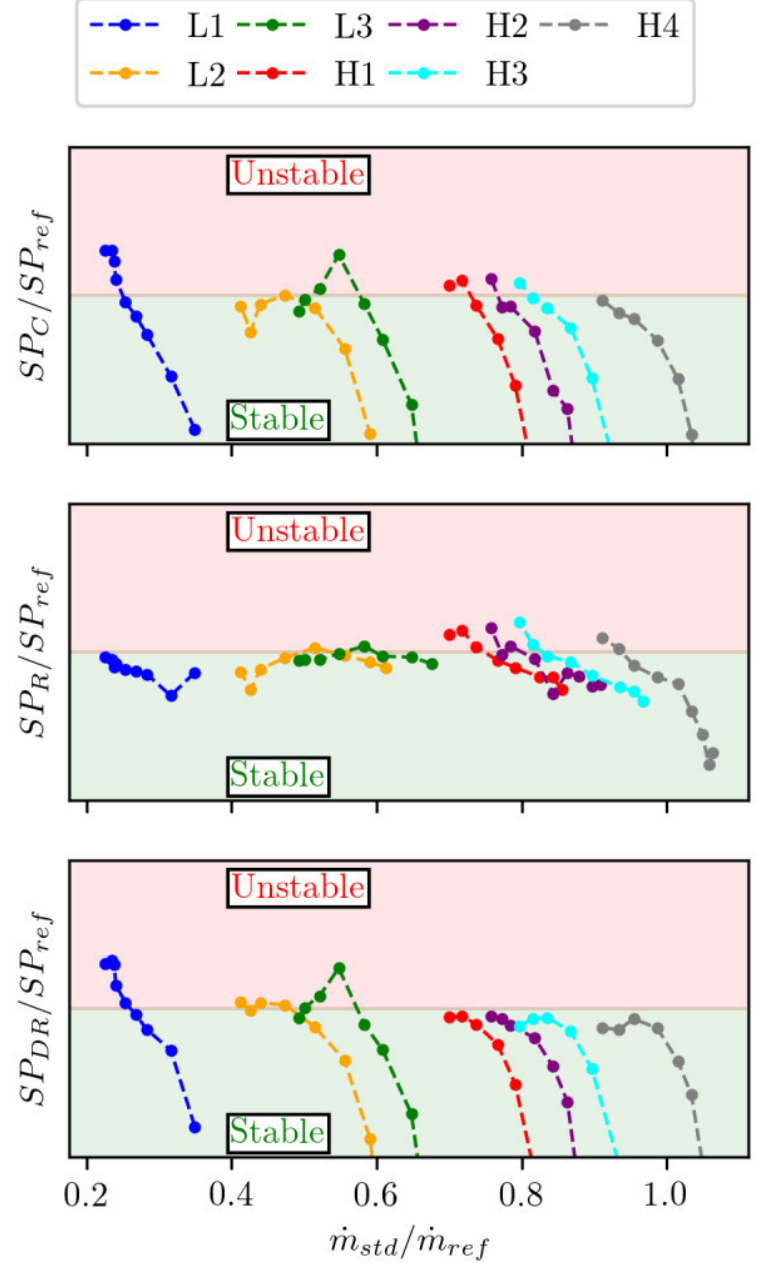

FIGURE 8: STABILITY PARAMETERS AT THE STAGGER ANGLE $0^{\circ}$

It is recalled that the stability parameter is only a first-order condition for stability of a compression system. However, it is clear that the radial diffuser is unstable whereas the impeller is stable at low rotation speeds. In contrast, at high rotation speeds, the radial diffuser is stable whereas the impeller is unstable. 
The stability parameters for a stagger angle of $20^{\circ}$ are plotted in Fig. 9.

$$
\begin{aligned}
& \rightarrow \text { L1 } \rightarrow-\text { L3 } \rightarrow-\text { H2 } \rightarrow-\text { H4 } \\
& \rightarrow \text { L2 } \rightarrow \text { H1 } \rightarrow \text { H3 }
\end{aligned}
$$
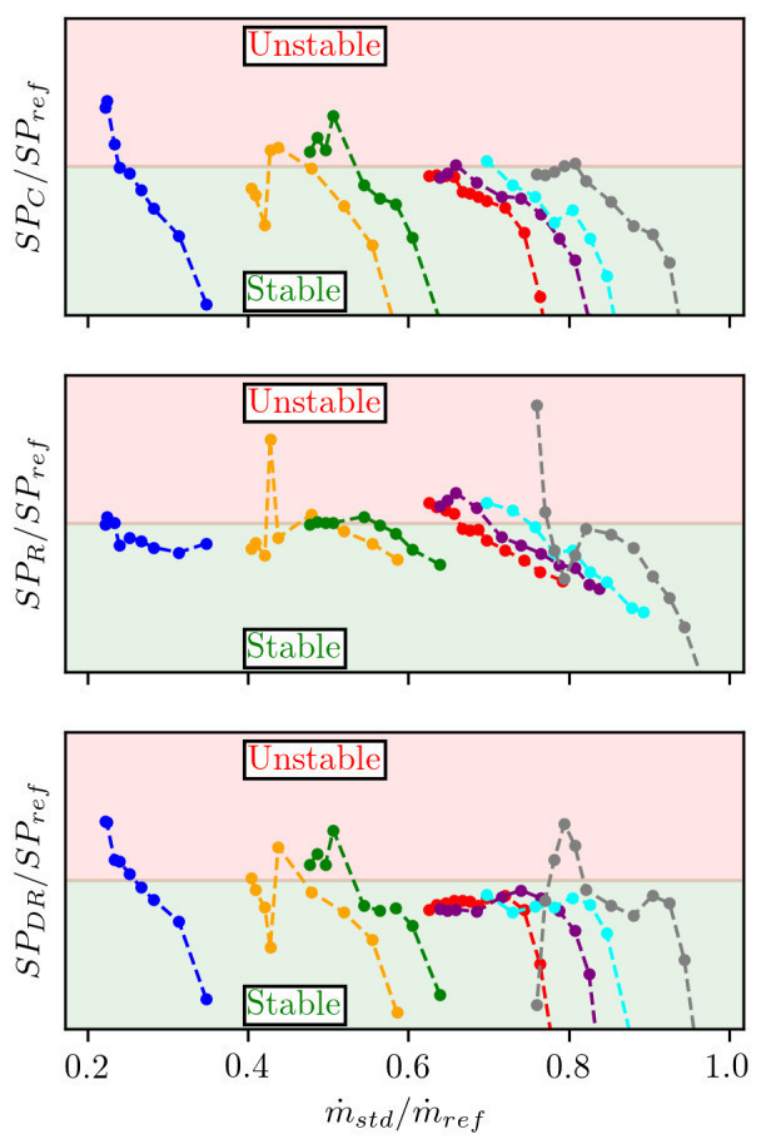

FIGURE 9: STABILITY PARAMETERS AT THE STAGGER ANGLE $20^{\circ}$

The trends between the low rotation speeds and the high rotation speeds observed at the stagger angle $0^{\circ}$ are repeated. Nevertheless, a difference should be mentioned: at the high rotation speed $\mathrm{H} 4$, the impeller becomes abruptly unstable near surge. The reasons are not yet understood.
The stability parameters for a stagger angle of $40^{\circ}$ are given in Fig. 10.

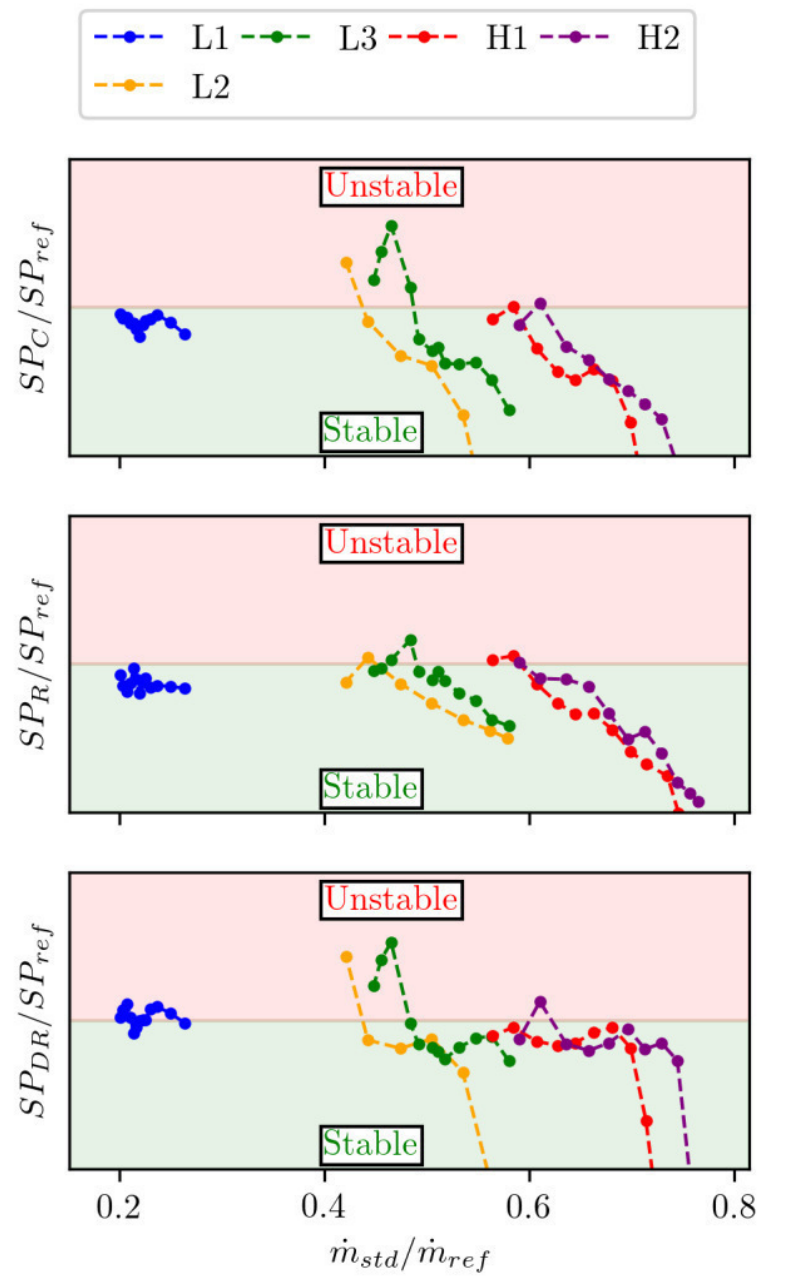

FIGURE 10: STABILITY PARAMETERS AT THE STAGGER ANGLE $40^{\circ}$

The trends at the low rotation speeds remain unchanged: the impeller is stable whereas the radial diffuser is unstable near deep surge. However, none of the components is unstable at high rotation speeds according to this first-order stability analysis. More accurate investigations (especially from unsteady pressure signals) will thus be carried out in order to understand the surge triggering at rotation speeds $\mathrm{H} 1$ and $\mathrm{H} 2$.

An overall similarity of the component stability at the 3 IGV stagger angles is observed. At low rotation speeds, the impeller is stable whereas the radial diffuser is unstable near surge. At high rotation speeds, it is the opposite configuration: the impeller is unstable and the radial diffuser is stable.

\section{DELAY OF THE ONSET OF ROTATING INSTABILITIES AT LOW ROTATION SPEEDS}

Rotating disturbances have been observed at the low rotation speed $\mathrm{L} 2$ on a large mass flow range at the stagger angle 
$0^{\circ}$ during previous work [15]. These rotating disturbances are in fact rotating instabilities according to the description of rotating instabilities proposed by Day [1]. These rotating instabilities are spatially consistent only during a few rotations of the impeller and their intensity and frequency are continuously varying, thus they differ from rotating stall.

The experimental signature of rotating instabilities at the 3 low rotation speeds is described below. The pressure fluctuations at 5 sensors circumferentially distributed at the shroud at the impeller inlet are plotted in Fig. 11 over one revolution at a stable operating point at low rotation speed L2. The phase average on 100 rotations is substracted in order to remove the synchronous phenomena such as the blade passing.

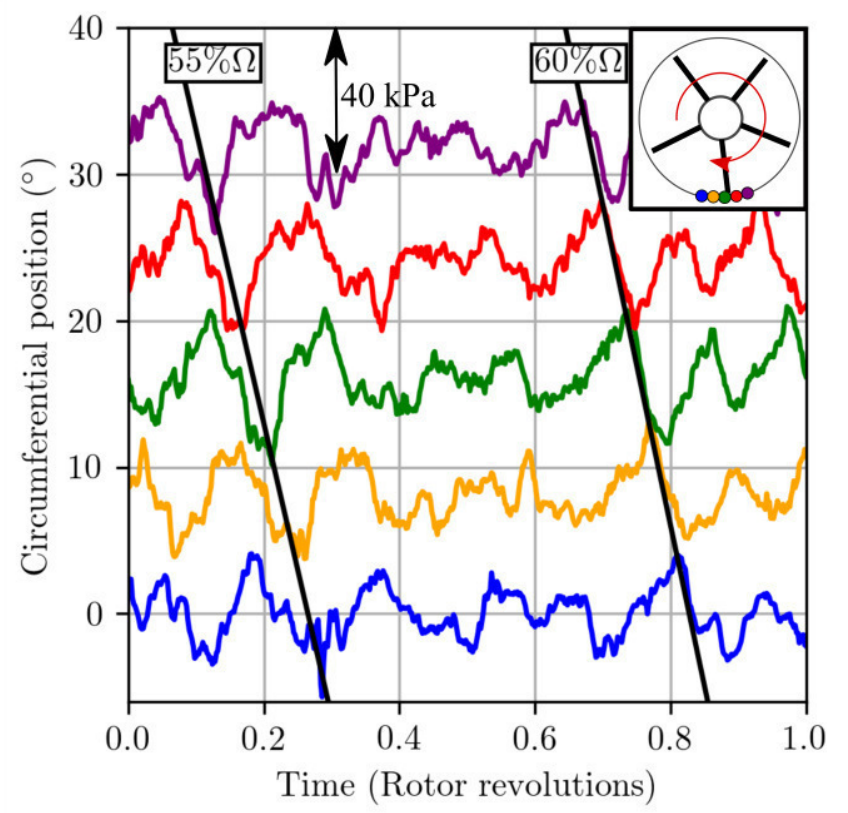

FIGURE 11: PRESSURE FLUCTUATIONS AT THE IMPELLER LEADING EDGE AT THE SHROUD AT ROTATION SPEED L2 AT THE STAGGER ANGLE $0^{\circ}$

It should be noted that even if only 5 sensors distributed on a small sector have been used for plotting Fig. 11 for clarity, the rotating instabilities are also observed when more sensors on a sector of about $60^{\circ}$ are considered.

The pressure fluctuations are significant: they reach up to $40 \mathrm{kPa}$. Some rotating instabilities can be identified: two are distinguished by black lines joining fluctuation peaks. Their rotating speed is between $55 \%$ and $60 \%$ of the rotor speed on this operating point.

The power spectral density of the pressure signal recorded by one sensor at the impeller leading edge at the shroud is also plotted in Fig. 12 for the same stable operating point. The power spectral density is also given for the choked operating point of the same rotation speed at the same stagger angle. At the operating point with rotating instabilities, the power is distributed on a bump which is centered on the rotating speed of the rotating instabilities. At the choked operating point, the power is only distributed in several peaks which are spaced of the rotation frequency. This characteristic of rotating instabilities is similar to the observations of Young et al. [2].

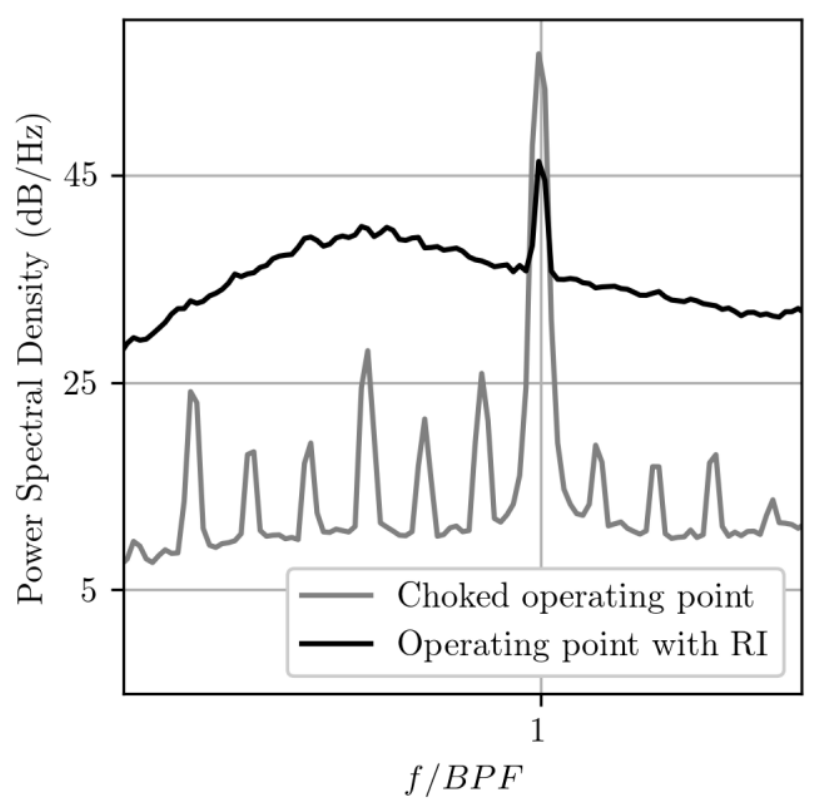

FIGURE 12: POWER SPECTRAL DENSITY OF PRESSURE FLUCTUATIONS AT THE IMPELLER LEADING EDGE AT THE SHROUD AT THE SAME OPERATING POINT

Rotating instabilities are detected by cross-correlating the pressure signals between the sensors. However, crosscorrelations on all the sensors distributed all around the circumference do not detect rotating instabilities due to their small spatial consistency. Therefore, cross-correlations are computed only between sensors distributed on a sector of about $60^{\circ}$.

This methodology is used at all stable operating points (previously presented). It is concluded that rotating instabilities also occur at low rotation speeds L1, L2 and L3 at the 3 stagger angles. The pressure fluctuations are the same order of magnitude at all low rotation speeds and at all stagger angles. In addition, the bump in the power spectral density has also the same shape between all operating points with rotating instabilities.

A critical value of the relative flow angle at the tip of the leading edge of the impeller $\beta_{1 \text {,critical }}{ }^{\text {tip }}$ is calculated by averaging the relative flow angle $\beta_{1}{ }^{\text {tip }}$ at which the rotating instabilities appear at each characteristic curve.

The operating points where rotating instabilities occur are identified by stars on Fig. 13. The x-axis is the standard mass flow rate and the $y$-axis is the ratio between the relative flow angle at the tip of the leading edge of the impeller $\beta_{1}{ }^{\text {tip }}$ and its critical value $\beta_{1 \text {,critical }}{ }^{\text {tip }}$. The change of the relative flow angle is consequent from choke to surge at a given rotation speed.

It should be noted that the relative flow angle is calculated by assuming that the meridional velocity is constant along the span. This assumption is obviously inaccurate but the bias introduced is equal between all rotation speeds and all stagger 
angles. The radial profile of the meridional velocity at the impeller inlet is extracted for a numerical operating point near the experimental operating point corresponding to the onset of rotating instabilities and plotted in Fig. 14 at IGV stagger angle of $0^{\circ}$. The radial profile of the meridional velocity is almost similar between the low rotation speeds. Therefore, if a mistake is done on the value of the critical flow angle, it does not depend on the rotation speed or stagger angle.

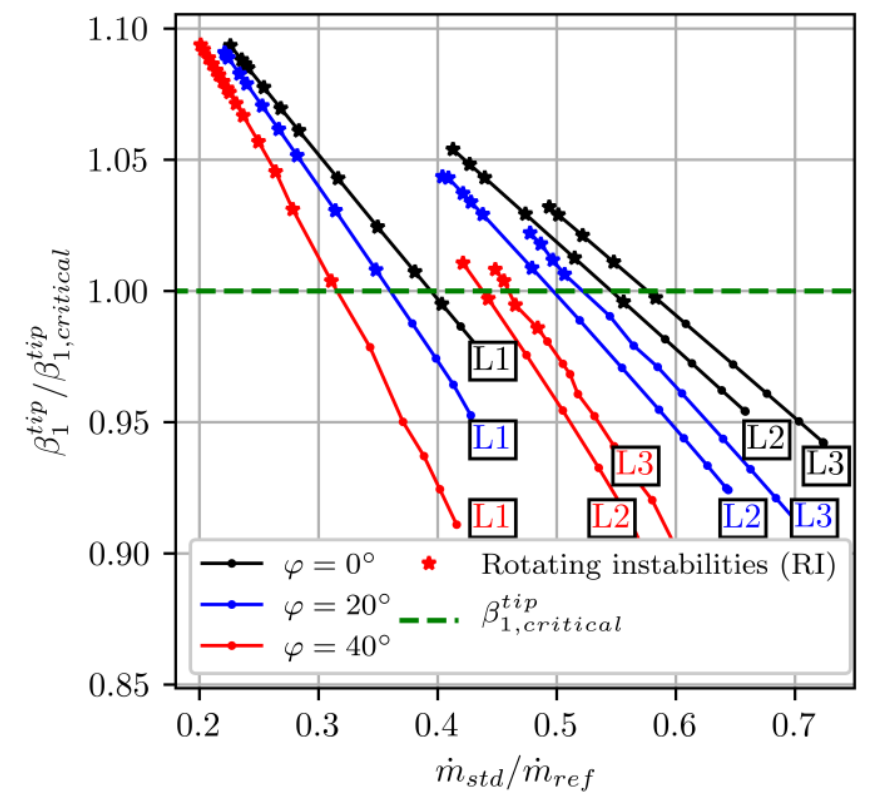

FIGURE 13: CORRELATION BETWEEN THE RELATIVE FLOW ANGLE AT THE IMPELLER INLET TIP AND THE ONSET OF ROTATING INSTABILITIES

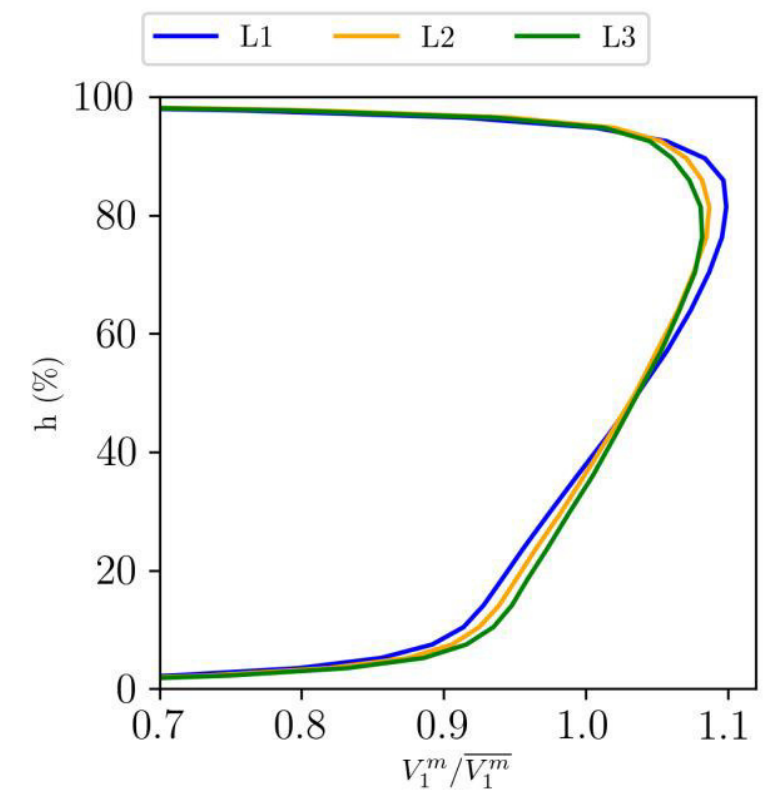

FIGURE 14: RADIAL PROFILE OF THE MERIDIONAL VELOCITY AT THE IMPELLER INLET AT IGV STAGGER ANGLE OF $0^{\circ}$
A strong correlation between the onset of rotating instabilities and the relative flow angle at the tip of the leading edge of the impeller $\beta_{1}{ }^{\text {tip }}$ is observed. The correlation is only proven false near deep surge at the rotation speed L3 at a stagger angle of $40^{\circ}$ (two operating points with RI before the critical relative flow angle on Fig. 13). This discrepancy might be due to mild surge observed at this rotation speed and as previously shown in Fig. 10, the radial diffuser is very unstable. Therefore, the flow is very unstable and it is not surprising that the correlation does not fit.

Rotating instabilities are not observed at high rotation speeds whatever the stagger angle. Indeed, the compressor stage surges before the relative flow angle at the impeller inlet reaches this critical value at high rotation speeds.

\section{INCREASE OF THE ALTERNATE RATE AT HIGH ROTATION SPEEDS}

Previous numerical and experimental studies [12],[13] at an IGV stagger angle of $0^{\circ}$ have shown that a steady alternate flow pattern settles in the radial diffuser over two channels at low mass flow rate on some rotation speeds. This steady alternate flow topology is also observed in RANS simulations at different IGV stagger angles (previously introduced). An example of static pressure maps obtained by RANS simulations is given for two mass flow rates in Fig. 15 a):

- Left column corresponds to the peak efficiency operating point. This flow topology is called "Periodic" because the flow is the same in each channel.

- $\quad$ Right column corresponds to an operating point at lower mass flow rate than the peak efficiency. This flow topology is called "Alternate" because it is very different over two adjacent channels: the left channel (channel 1) is stalled whereas the right channel (channel 2) is free. This flow pattern is steady in time and space.

It should be noted that these numerical flow fields are only given for an illustrative purpose and not for a comparison between experimental and numerical results.

Well discretized pressure maps are not available experimentally. An indicator called the "Alternate rate" (A) using the steady pressure profiles measured at the radial diffuser inlet is developed in order to estimate how much the topology is alternate. The diffuser inlet is chosen because the sensors are the most numerous there (see Fig. 2) and the flow difference between two adjacent channels is the most pronounced. A similar indicator is presented in [13] but the methodology of calculation is considerably simplified in the present work. The alternate rate $\mathrm{A}$ compares the difference between the circumferential static pressure profiles at the diffuser inlet between the channel 1 and channel 2 :

$A=\frac{1}{\theta_{D R} \overline{P_{\text {chan }, 1+2}}} \int_{0}^{\theta_{D R}}\left|P_{\text {chan } .1}(\theta)-P_{\text {chan }, 2}(\theta)\right| d \theta$ 


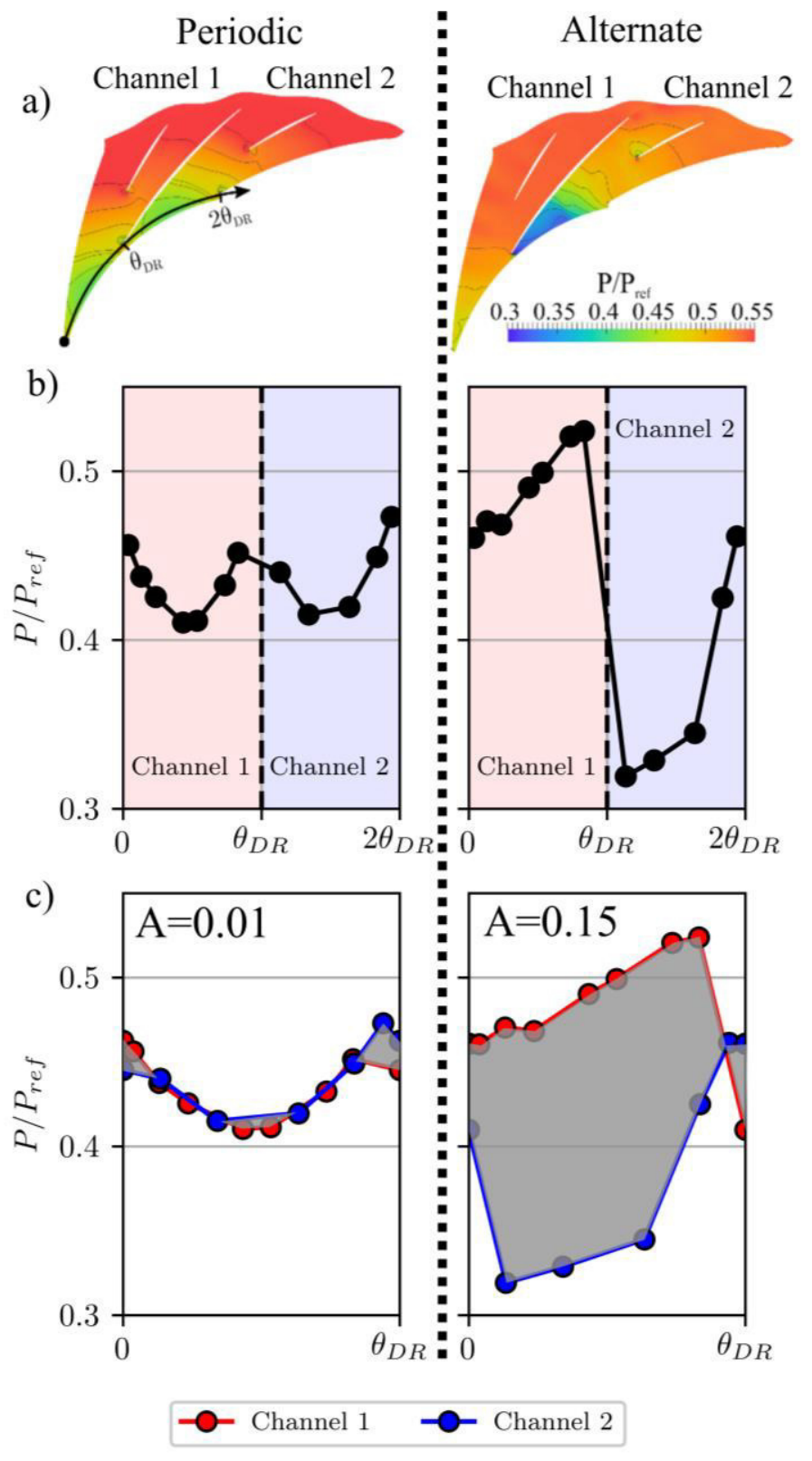

FIGURE 15: EXAMPLE OF CALCULATION OF THE ALTERNATE RATE WITH NUMERICAL RESULTS (a: STATIC PRESSURE MAP b: PRESSURE PROFILE AT THE DIFFUSER INLET, c: COMPARISON OF PRESSURE PROFILES BETWEEN THE TWO CHANNELS, LEFT COLUMN: PERIODIC FLOW, RIGHT COLUMN: ALTERNATE FLOW)

The alternate rate $\mathrm{A}$ is actually calculated from discrete values with a trapezoids integration. An example from numerical results in a high alternate case is given to illustrate the methodology. The numerical flow fields are probed like in the experiments. The circumferential pressure profiles probed of the numerical flow fields are plotted in Fig. 15 b for both flow topologies. The pressure profiles at the inlet of channel 1 and channel 2 are compared in Fig. $15 \mathrm{c}$ : the alternate rate corresponds to the area shaded between the two pressure profiles.

The alternate rate is 0.01 in the periodic case, it is not zero because there are differences of discretization between the two channels. In the alternate case, the alternate rate is 0.15 . Therefore, the indicator A discriminates well the two flow topologies because there is a ratio of 15 between the two cases.

The values of A are then calculated from the experimental pressure data at rotation speed $\mathrm{H} 2$ in order to study the impact of the IGV stagger angle on the alternate rate. The alternate rate $\mathrm{A}$ is plotted against the standard mass flow rate from the peak efficiency operating point to the last stable operating point in Fig. 16. The alternate rate increases towards surge for the three stagger angles. Moreover the alternate rate near surge is higher at stagger angles of $40^{\circ}$ and $20^{\circ}$ than at stagger angle of $0^{\circ}$.

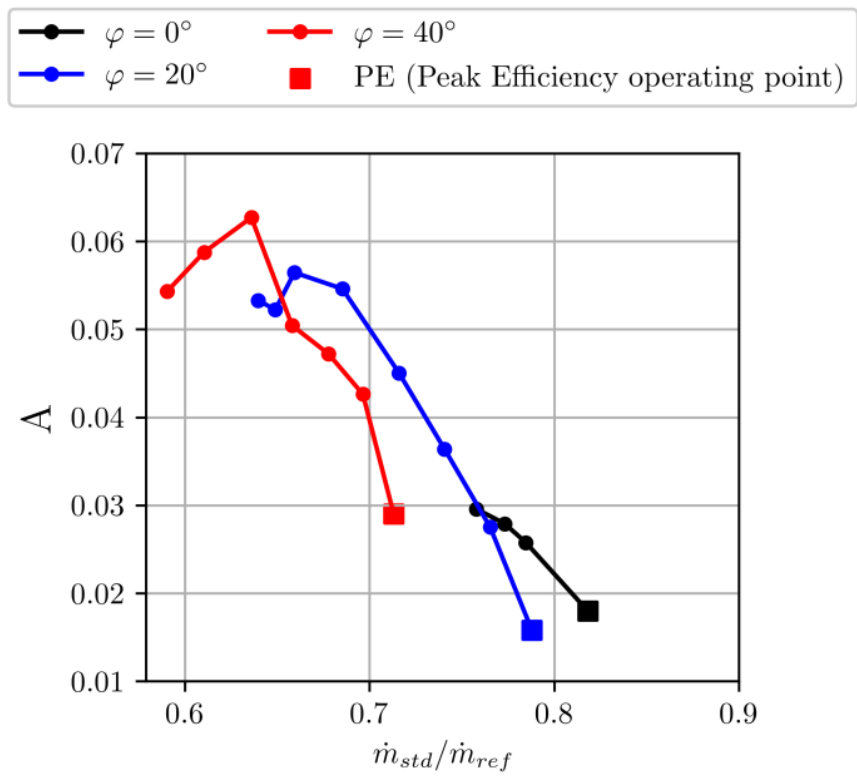

FIGURE 16: EVOLUTION OF ALTERNATE RATE FROM PEAK EFFICIENCY OPERATING POINT TO LAST STABLE OPERATING POINT AT ROTATION SPEED H2 FOR 3 IGV STAGGER ANGLES

This difference of alternate rate at the last stable operating point between the IGV stagger angle is investigated. The alternate rate $\mathrm{A}$ against the difference between the absolute flow angle at the radial diffuser inlet and the camber angle $\Theta_{\mathrm{C}}$ is plotted in Fig. 17. The absolute flow angle is calculated by using Euler's equation and the mass flow rate conservation. Aside from the two last operating points near surge, a satisfactory correlation is observed between the alternate rate and the absolute flow angle. Therefore, the difference of alternate rate at last stable operating point between stagger angles is explained by a difference of absolute flow angle at the diffuser inlet. 

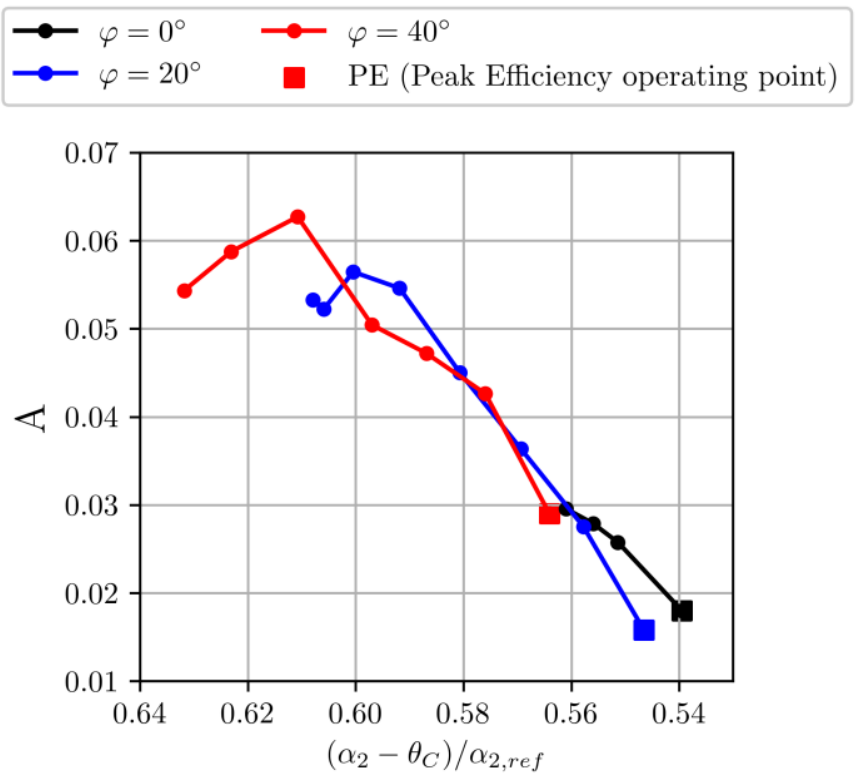

FIGURE 17: CORRELATION BETWEEN THE ALTERNATE RATE AND THE ABSOLUTE FLOW ANGLE AT THE RADIAL DIFFUSER INLET AT ROTATION SPEED H2

The unsteady alternate rate is also calculated with the same methodology but with the unsteady pressure sensors. The standard deviation of the temporal fluctuations of the alternate rate remains very low for all operating points at rotation speed $\mathrm{H} 2$. Therefore, the alternate flow pattern is fully steady and is not coupled with a mode.

Finally, the steady alternate rate is calculated for all high rotation speeds and it is observed that there is a large zone of high alternate rate $(\mathrm{A}>0.03)$ ranging from rotation speed $\mathrm{L} 3$ to rotation speed $\mathrm{H} 4$ beyond the surge line at IGV stagger angle of $0^{\circ}$ in Fig. 18. The right boundary of this zone of high alternate rate corresponds to a critical absolute flow angle at the radial diffuser inlet.

\section{CONCLUSION}

A research aeronautical centrifugal compressor stage is tested at 3 IGV stagger angles. It is proven that humidity should be taken into account for calculating the performance. A nonlinear effect of the stagger angle on the choked mass flow is observed. It is also observed that the shift of the surge line as the IGV stagger angle increases highly depends on the rotation speed.

A first-order stability analysis of the impeller and diffuser sub-components showed that the diffuser (resp. impeller) is the first unstable component at low (resp. high) rotation speeds. This situation is unaltered by increasing the IGV stagger angle.

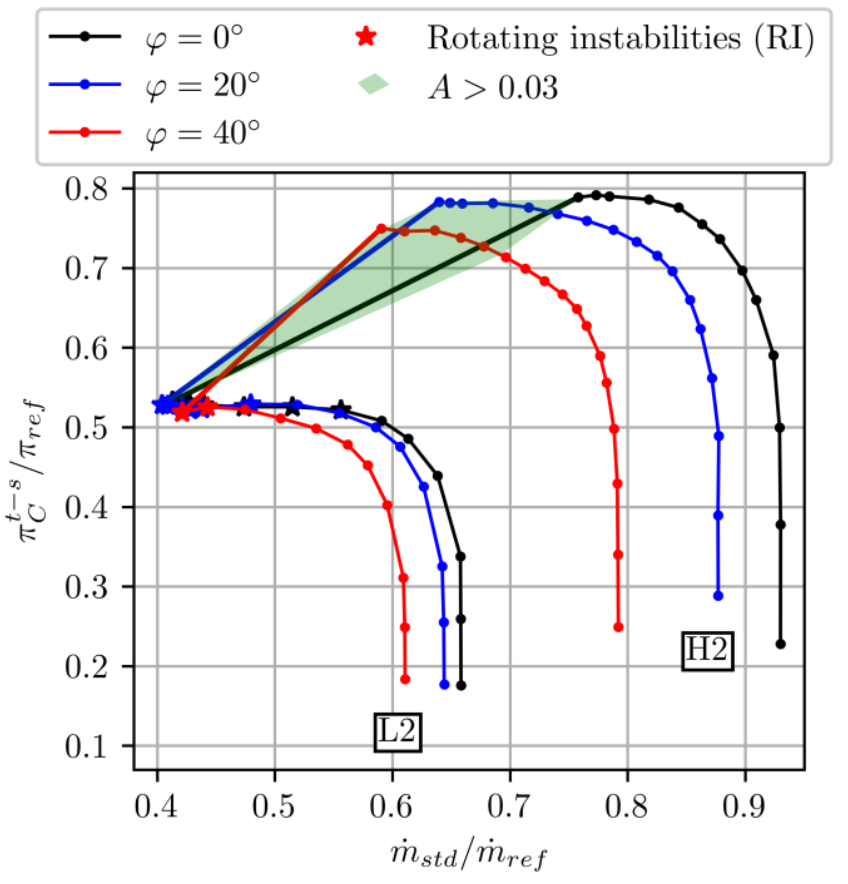

FIGURE 18: ZONE OF HIGH ALTERNATE RATE BEYOND THE SURGE LINE AT STAGGER ANGLE OF $0^{\circ}$

Rotating instabilities at the impeller inlet are observed at low rotation speeds at each IGV stagger angle. It is shown that their occurrence is conditioned by the relative flow angle at the tip of the leading edge of the impeller. As the IGV stagger angle increases, the mass flow decreases to maintain a given inlet relative flow angle. As a result, the onset of the rotating instabilities is delayed towards lower mass flow rates.

At high rotation speeds, the absolute flow angle at the diffuser inlet near surge decreases as the IGV stagger angle increases. Therefore, the flow is highly alternate over two adjacent channels of the radial diffuser (one channel stalled and one channel free) beyond the surge line at IGV stagger angle of $0^{\circ}$. Indeed, there is a critical absolute flow angle below which the flow is highly alternate over two channels in the diffuser.

\section{ACKNOWLEDGEMENTS}

The authors would like to thank Pierre Laucher, Sebastien Goguey, Gilbert Halter and Benoit Paoletti for manufacturing the test bench and supporting the measurements.

The authors would like to thank also Safran Helicopter Engines which supported this study. This project has received funding from the Clean Sky 2 Joint Undertaking under the European Union's Horizon 2020 research and innovation program under grant agreement $\mathrm{N}^{\circ} 820099$.

\section{NOMENCLATURE}

$\begin{array}{ll}\dot{m} & \text { Mass flow } \\ \mathrm{h} & \text { Span height } \\ \mathrm{r} & \text { Specific gas constant } \\ \text { APU } & \text { Auxiliary Power Unit }\end{array}$




$\begin{array}{ll}\text { BPF } & \text { Blade Passing Frequency } \\ \text { IGV } & \text { Inlet Guide Vanes } \\ P & \text { Pressure } \\ \mathrm{P}_{\mathrm{H}_{2} \mathrm{O}} & \text { Partial pressure of water vapor } \\ \mathrm{P}^{*}{ }_{\mathrm{H} 2 \mathrm{O}} & \text { Equilibrium vapor pressure } \\ \text { RANS } & \text { Reynolds-Averaged Navier-Stokes } \\ \text { RI } & \text { Rotating instabilities } \\ \mathrm{SP} & \text { Stability parameter } \\ \mathrm{T} & \text { Temperature } \\ \mathrm{U} & \text { Peripheral velocity } \\ \text { V } & \text { Absolute velocity } \\ \text { W } & \text { Relative velocity }\end{array}$

\section{Greek Symbols}

$\begin{array}{cl}\alpha & \text { Absolute flow angle } \\ \beta & \text { Relative flow angle } \\ \gamma & \text { Specific heat ratio } \\ \eta & \text { Isentropic efficiency } \\ \pi & \text { Pressure ratio } \\ \varphi & \text { IGV stagger angle } \\ \Phi & \text { Relative humidity }\left(\mathrm{P}_{\mathrm{H} 2 \mathrm{O}} / \mathrm{P}^{*}{ }_{\mathrm{H} 2 \mathrm{O}}\right) \\ \Omega & \text { Rotation speed } \\ \Theta_{\mathrm{C}} & \text { Camber angle } \\ \text { Subscripts } & \\ \text { chan } & \text { Channel } \\ \mathrm{C} & \text { Compressor }(\mathrm{IGV}+\mathrm{IMP} .+\mathrm{RD}) \\ \mathrm{IMP} . \text { or } \mathrm{R} & \text { Impeller } \\ \mathrm{RD} & \text { Radial Diffuser } \\ \text { ref } & \text { Reference }(\text { value }) \\ \text { std } & \text { Standard } \\ 0 & \text { IGV inlet } \\ 1 & \text { IMP. inlet } \\ 2 & \text { RD inlet } \\ 3 & \text { RD outlet } \\ \text { Superscripts } & \\ \mathrm{m} & \text { Meridional } \\ \mathrm{s} & \text { Static } \\ \mathrm{t} & \text { Total } \\ \text { s-s } & \text { Static to static } \\ \mathrm{t}-\mathrm{s} & \text { Total to static } \\ \mathrm{t}-\mathrm{t} & \text { Total to total } \\ & \end{array}$

\section{REFERENCES}

[1] Day, I. J., 2016, "Stall, surge, and 75 years of research", Journal of Turbomachinery, 138(1): 011001.

[2] Young, A., Day, I., Pullan, G., 2012, "Stall warning by blade pressure signature analysis", Journal of Turbomachinery, 135(1): 011033.

[3] Schwerdt, L., Willeke, T., Huxdorf, O., Krone, J. H., Friedrichs, J., Riemenschneider, J., Monner, H. P., Seume, J. R., 2019, "Active flow control in an axial compressor by zero-netmass-flux actuation", In International Symposium on Experimental Computational Aerothermodynamics of Internal Flows.

[4] Van den Braembussche, R., 2018, Design and Analysis of Centrifugal Compressors, John Wiley and Sons, NY.
[5] Whitfield, A., Abdullah, A. H., 1997, "The performance of a centrifugal compressor with high inlet prewhirl”, In International Gas Turbine \& Aeroengine Congress \& Exhibition, American Society of Mechanical Engineers.

[6] Rodgers, C., 1991, “Centrifugal compressor inlet guide vanes for increased surge margin”, Journal of Turbomachinery, 113, pp. 696-702.

[7] Simon, H., Wallmann, T., Mönk, T., 1987, "Improvements in performance characteristics of single-stage and multistage centrifugal compressors by simultaneous adjustments of inlet guide vanes and diffuser vanes", Journal of Turbomachinery, 109, pp. 41-47.

[8] Ishino, M., Iwakiri, Y., I., Bessho, A., Uchida, H., 1999, "Effects of variable inlet guide vanes on small centrifugal compressor performance", In International Gas Turbine \& Aeroengine Congress \& Exhibition, American Society of Mechanical Engineers.

[9] Tian, Y., Tang, Y., Wang, Z., Xi, G., "Influence of adjustable inlet guide vanes on the performance characteristics of a shrouded centrifugal compressor", In ASME Turbo Expo 2017: Turbomachinery Technical Conference and Exposition, American Society of Mechanical Engineers.

[10] Mohseni, A., Goldhahn, E., Van den Braembussche, R. A., Seume, J. R., "Novel IGV designs for centrifugal compressors and their interaction with the impeller", In ASME Turbo Expo 2012: Turbomachinery Technical Conference and Exposition, American Society of Mechanical Engineers.

[11] Uchida, H., Kashimoto, A., Iwakiri, Y., 2006, "Development of wide flow range compressor with variable inlet guide vane", R\&D Review of Toyota CRDL, 41(3), pp. 9-14.

[12] Benichou, E., Trébinjac, I, "Numerical analysis of an alternate stall in a radial vaned diffuser", In ASME Turbo Expo 2016: Turbomachinery Technical Conference and Exposition, American Society of Mechanical Engineers.

[13] Moënne-Loccoz, V., Trébinjac, I., Poujol, N., Duquesne, P., 2020, "Detection and analysis of an alternate flow pattern in a radial vaned diffuser", Int. J. Turbomach. Propuls. Power, 5(1).

[14] Glück, B., 1991, "Zustands-und stoffwerte für wasser, dampf und luft; verbrennungstechnik, verlag für bauwesen", Berlin.

[15] Moënne-Loccoz, V., Trébinjac, I., Benichou, E., Goguey, S., Paoletti, B., and Laucher, P., 2017, "An experimental description of the flow in a centrifugal compressor from alternate stall to surge", Journal of Thermal Science, 26(4), pp. 289-296. [16] Dean, R. C., 1974, "The fluid dynamic design of advanced centrifugal compressors", Advanced Radial Compressors, Inst. For Fluid Dyn. 


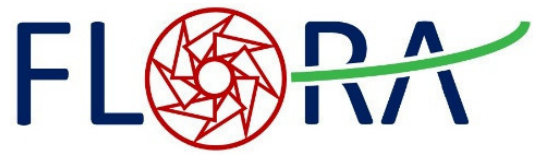

The presented research was supported through Clean Sky 2 Joint Undertaking (JU), project FLORA under grant agreement $\mathrm{N} \times 820099$. The JU receives support from the European Union's Horizon 2020 research and innovation programme and the Clean Sky $2 \mathrm{JU}$ members other than the Union. This publication reflects only the author's view and the JU is not responsible for any use that may be made of the information it contains.

https://www.researchgate.net/project/FLORA-FLOw-control-in-RAdial-compressor

Pierre.duquesne@ec-lyon.fr
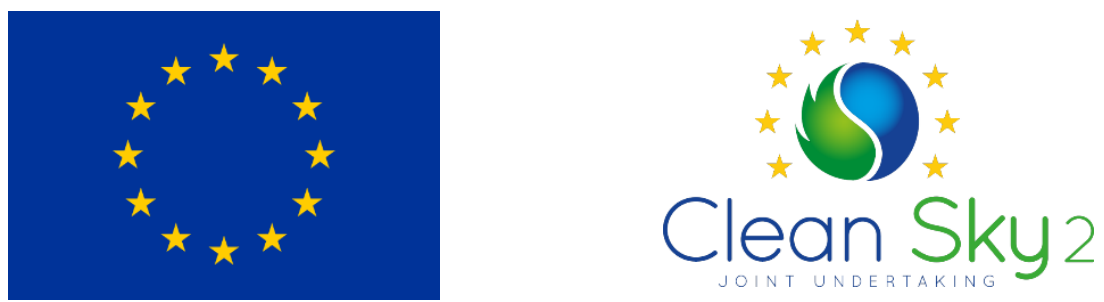